\title{
Numerical and experimental investigation of (de)lithiation-induced strains of bicontinuous silicon- coated nickel inverse opal anodes
}

\author{
Hoon-Hwe Cho ${ }^{\mathrm{a}}$, Matthew P. B. Glazer ${ }^{\mathrm{a}}$, Qian Xub ${ }^{\mathrm{b}}$, Heung Nam $\mathrm{Han}^{\mathrm{c}}$ and David C. \\ Dunand $^{\mathrm{a}^{*}}$ \\ ${ }^{\mathrm{a}}$ Department of Materials Science and Engineering, Northwestern University, 2220 \\ Campus Drive, Evanston, IL, 60208, USA \\ ${ }^{\mathrm{b}}$ Waikato Centre for Advanced Materials (WaiCAM), School of Engineering, University \\ of Waikato, Hamilton, New Zealand \\ (currently at Surgionix Ltd., Private Bag 305 543, Auckland, New Zealand) \\ ${ }^{\mathrm{c}}$ Department of Materials Science \& Engineering and Center for Iron \& Steel Research, \\ RIAM, Seoul National University, Seoul, 151-744, Republic of Korea
}

*Corresponding author: Prof. David C. Dunand Email: dunand@northwestern.edu 


\section{Abstract}

A volume expansion of up to $\sim 310 \%$ occurs upon the lithiation of silicon in Si-coated nickel inverse opal anodes, which causes (de)lithiation-induced mismatch stresses and strains between the Si and Ni during battery cyclical (dis)charging. These (de)lithiationinduced mismatch strains and stresses are modeled via sequentially coupled diffusionand stress-based finite element (FE) analysis, which takes the mechanical contact between the Si and Ni phases into account, as well as the complex geometry and material properties of the Si-coated Ni inverse opal anode system. During lithiation, compressive strains are developed in the $\mathrm{Ni}$ scaffold since the $\mathrm{Si}$ active layer expands. A rapid recovery of these lithiation-induced mismatch strains occurs during subsequent delithiation, though full recovery is not achieved. Strain histories upon multiple (de)lithiation cycles vary with the choice of various mechanical contact conditions employed between the two phases, since the mechanical contact properties determine how the contacted phases interact mechanically. The numerically predicted strains are compared with experimental strain data collected in operando using X-ray diffraction. The simulated strain histories agree with the measured data, enabling the possibility of predicting mechanical performance and eventual degradation using only numerical modeling. In particular, the FE model indicates that plastic deformation occurs first in the lithiated Si active layer, then in the Ni scaffold.

Keywords: silicon anodes, lithiation strain, diffusion-stress coupling, mechanical contact, $X$-ray diffraction $(X R D)$ 


\section{Introduction}

Understanding lithium ion insertion-induced volume changes in lithium-ion batteries (LIBs) is important, since those can affect mechanical stability in LIBs. Silicon is a very promising anode material due to its high gravimetric capacity (up to $3580 \mathrm{mAhg}^{-1}$ ) [1], but it suffers from very high volume change (>300\%) during lithiation. This lithiationinduced volume change can result in large mechanical stresses, leading to damage of silicon-based anodes through delamination, cracking and pulverization, and thereby causing a rapid battery capacity fade [2]. Much effort has been devoted to exploring the fundamental nature of this volume change by employing experimental approaches, such as electron microscopy [3-6], atomic force microscopy [7], optical cantilever measurements such as multi-beam optical sensor techniques [8, 9], and X-ray diffraction (XRD) [10-14], which are only useful to examine the microstructural and geometric characteristics of anode due to the lithiation-induced volume change. However, considerably less attention has been given to understanding the complicated interactions among the various mechanical and diffusional phenomena, which occur simultaneously during lithiation process. Finite element (FE) analysis [15, 16] enables a deeper understanding of the lithiation process, and a FE model coupled with experimental results is a powerful tool for exploring this complex process.

Consequently, to investigate the lithium diffusion-induced volume change and the corresponding mechanical damage, we present here an FE analysis tool which takes into account the complicated geometry and precise material properties of the Si-coated $\mathrm{Ni}$ inverse opal anode system [17] and the types of mechanical contacts between the Si and Ni phases. The computed strain data are compared with in operando XRD-based strain 
measurements to verify the validity of the FE model, demonstrating that a combination of different approaches can be used to predict the mechanical behavior of the anode due to the lithiation-induced volume change through elasto-plastic deformation. XRD-based strain measurement techniques were previously utilized to describe average strains, axial strains, deviatoric strains and strain distributions, as well as multi-phase strain partitioning in a variety of materials systems [18-25]. XRD techniques are able to nondestructively measure both phase composition and the distribution of strains in bulk material quantities and buried interfaces in sensitive sample environments, which can be extremely difficult to achieve using other characterization techniques. Recently, Yang et al. and others have used XRD to characterize the transition between crystalline and amorphous Si during the first lithiation cycle as well as the crystallization of amorphous lithiated Si at high degrees of lithiation [11]. However, at size regimes below $\sim 2 \mu \mathrm{m}$ in nanostructured anodes employing amorphous $\mathrm{Si}$, there is no clear formation of the $\mathrm{Li}_{15} \mathrm{Si}_{4}$ phase [11]. The amorphous nature of nanostructured Si during (de)lithiation cycling precludes the use of XRD to directly measure strains in the $\mathrm{Si}$ and thus requires indirect measurement techniques to quantify the strain present in the Si during cycling. Therefore, in the amorphous Si-coated crystalline structure anodes, only the strain developed in the crystalline structure can be measured by XRD to explore the mechanical behavior of anodes during lithiation, indicating the importance of FE model which can predict strains in both the Si and the crystalline structure.

Several FE models employing rigorous constitutive laws and governing equations have been developed to describe the lithiation behavior and mechanics of anodes. Bower and Guduru have developed a simple FE model to investigate the coupled processes of 
diffusion, deformation, and fracture in the LIBs [15]. More recently, Liu presented a FE model taking into account the coupling effect between diffusion and stress along with the dependence on the lithium concentration of elastic properties of the active material [16]. However, considerably less attention has been given to combining numerical models with experimental measurements despite the significant and practical importance of this approach. Comparisons between FE models and empirical data can be utilized not only to validate model behavior, but also to inform which primary mechanical degradation mechanisms may contribute to capacity fade and failure in an anode system. These comparisons may also enable the effective and rapid design of new anode composites and structures with greater capacities and lifetimes through a better understanding of stress concentrations, plasticity and mechanical failure.

In this work, we aim at combining sequentially-coupled diffusion and stress FE modeling with in operando XRD-based strain measurements for an anode system consisting of amorphous silicon on a nickel inverse opal substrate [17, 26]. Our FE model incorporates various mechanical contact conditions between the two phases in the anode (amorphous $\mathrm{Si}$ and $\mathrm{Ni}$ ), the complex, bicontinuous geometry of the inverse opal system, and precise mechanical properties for both phases. Strains developed in both the Si active layer and the Ni scaffold due to the volume changes associated with the (de)lithiation of the Si active layer are computed using the FE model. The predicted values are then compared with the measured in operando strains based on the $\mathrm{Ni}$ average lattice crystallographic spacings. Additionally, the effects of different mechanical contact conditions between two phases on these internal strains are examined by introducing extreme contact conditions such as no-interaction condition between the contacted 
surfaces and no-volume change of the Ni scaffold while keeping volumetric strain of the Si active layer unchanged, since the mechanical contact properties determines how the contacted phases interact mechanically via load transfer.

\section{Computational Model and Methodology}

\subsection{Governing equations}

We consider the insertion of solute atoms into a solid. During charging, Li atoms are inserted into the anode by electrochemical reaction occurring at the surface of the solid, which leads to volume expansion and mechanical stress. In the following, we will summarize the governing equations of deformation and diffusion in the electrode. It is also assumed that the developed strain is small and thus the mechanics of infinitesimal straining can be applied.

The constitutive equation for diffusion-induced deformation of an elastic solid can be expressed as [16, 27, 28]:

$\varepsilon_{i j}=\frac{1}{E}\left[(1+v) \sigma_{i j}-v \sigma_{k k} \delta_{i j}\right]+\frac{c \Omega}{3} \delta_{i j}$

where $\varepsilon_{i j}(i, j=1,2,3)$ are the components of strain tensors, $\sigma_{i j}$ are the components of stress tensors, $\delta_{i j}$ is the Kronecker delta, $c$ is the concentration of the diffusing component ( $\mathrm{mol} / \mathrm{m}^{3}$ ), $\Omega$ is the coefficient of the volume expansion per mole of solute atoms $\left(\mathrm{m}^{3} / \mathrm{mol}\right)$, and $E$ and $v$ are Young's modulus and Poisson's ratio, repectively. The last three parameters are assumed constant, independent of $c$. The volumetric expansion of Si during lithiation is described in detail in the supplementary material.

The stress increment of Cauchy stress $d \sigma$ can be expressed as: 
$d \sigma=C^{e}: d \varepsilon^{e}$

where $C^{e}$ and $d \varepsilon^{e}$ are the elastic stiffness tensor and elastic strain increment, respectively. The total strain increment $d \varepsilon^{T}$ is taken as:

$d \varepsilon^{T}=d \varepsilon^{e}+d \varepsilon^{d}+d \varepsilon^{p}$

where $d \varepsilon^{d}$ and $d \varepsilon^{p}$ are the strain increments due to diffusion and the plastic strain increment, respectively. Consequently, the stress increment becomes:

$d \sigma=C^{e}:\left(d^{T}-d \varepsilon^{d}-d \varepsilon^{P}\right)$

Li diffusion through the electrode is driven by a chemical potential $\mu$ [15]:

$\mu=\mu_{0}+R \ln c$

where $\mu_{0}$ is a constant, $R$ is the gas constant, and $T$ is temperature. The hydrostatic stress effect on the Li diffusion model is neglected, which will be explained on the subsequent diffusion flux equation. The molar flux of Li crossing a unit area $J_{i}$ is related to the chemical potential by [15]:

$$
J_{i}=-\frac{D(T) \rho_{0} c}{R T} \frac{\partial \mu}{\partial x_{i}}
$$

where $D$ is a temperature-dependent diffusion coefficient, $\rho_{0}$ is the density of the anode material prior to lithiation, and $x$ is a position vector. The effect of pressure on diffusion $[29,30]$ is neglected in this work, which can be justified by the relatively low charging 
rates, reduced diffusion length in the nanostructured material, and the relatively high diffusivity of $\mathrm{Li}$ in Si. The pressure gradient effect on the diffusion flux during lithiation is described in detail in the supplementary material. The evolution equations for $\mathrm{Li}$ concentration are completed by the mass conservation equation [15]:

$\rho_{0} \frac{\partial c}{\partial t}+\frac{\partial J_{i}}{\partial x_{i}}=0$

where $t$ is the time.

\subsection{Finite element modeling}

The governing equations listed in the previous section are implemented in the ABAQUS/Standard software by incorporating a user-defined subroutine. In this FE model, the equations for diffusion and mechanical deformation are solved sequentially, which means that $\mathrm{Li}$ concentrations extracted from the diffusion computation are subsequently passed to the mechanical deformation computation.

The representative volume element (RVE) of the Si-coated Ni inverse opal electrode, which is reduced into a one-eighth sub-cell, using the symmetry from the periodic cubic unit cell, was used to compute mechanical deformation and diffusion during (de)lithiation, as illustrated in Fig. 1. The RVE consists of both Si active layer and Ni scaffold, which are in mechanical contact during the process. The active layer has a thickness of $30 \mathrm{~nm}$ [31], and the pore fraction and size of the RVE are $50 \%$ and $500 \mathrm{~nm}$, respectively. The RVE has 9,759 tetrahedral elements consisting of Si (9,063 elements) and Ni (696 elements), as shown in Fig. 1. The inverse opal Ni scaffold can be conceptually understood to be a network of nodes and struts which comprise 40 and $60 \%$ of the $\mathrm{Ni}$ 
volume, respectively [26]. The volume fractions of Ni scaffold and Si active layer are 26 and $24 \%$ of the total volume of the structure, respectively.

The boundary conditions resulting from the electrochemical reactions are enforced in the RVE. The initial condition is $c=0$ at $t=0$ at all places. During operation, a Li-ion concentration, $c_{s}$, on the electrode surface can be determined from the electrode voltage, so the concentration of Li-ion on the surface during lithiation is set as $c=c_{s}$ on the surface of the electrode for lithiation. The maximum concentration of Li-ion, $c_{\mathrm{m} \text { a }}$, is 54.00 lithium ions $/ \mathrm{nm}^{3}$ [32]. The electric charge is $0.5 \mathrm{C}$, which results in a Li flux (Eq. 6) on the surface of the electrode. For delithiation, the simulation starts with the fully lithiated state. Since the discharging rate is the same as the charging rate, the flux boundary condition is simply adjusted from in-flux to out-flux with the same magnitude. The RVE has mirror boundary conditions along $x, y$ and $z$ directions to enforce periodic boundary conditions.

The Si active layer and Ni passive scaffold are in contact, which can be specified by the normal and tangential behavior of the contact. For the normal behavior, the contact pressure increases linearly from the moment of contact between the two surfaces while keeping no inter-penetration between the contacted surfaces (Fig. 2). The linear contact behavior between the two surfaces is described in detail in the supplementary material. We assumed that there is no slip between the contacted surfaces for the tangential behavior (Fig. 2). To investigate the effect of the mechanical contact condition on the mechanical deformation, we explore extreme contact conditions: (1) no interaction 
condition between the contacted surfaces and (2) no volume change of the Ni scaffold while keeping volumetric strain of the Si active layer (Fig. 2).

The materials properties of $\mathrm{Si}$ and $\mathrm{Ni}$ and parameter values used in the simulation are listed in Table 1.

\section{Results and Discussion}

\subsection{Strain predictions during (de)lithiation process}

Fig. 3a shows the computed $\mathrm{Li}$ concentration (black) and the corresponding volumetric strain (blue) profiles in $\mathrm{Si}$ upon the first lithiation-delithiation cycle. The $\mathrm{Li}$ concentration increases linearly upon lithiation due to the Li flux into the electrode, and the average molar content of $\mathrm{Li}$ in $\mathrm{Si}$ become 3.77 moles $\mathrm{Li}$ per mole Si at full lithiation. The increase of Li concentration causes a volume change of $\mathrm{Si}$ up to $310 \%$, as shown in the Si model in the middle of Fig. 3b. The computed Li concentration distribution at the full lithiation stage shows that the $\mathrm{Li}$ ions diffuse into the $\mathrm{Si}$ on lithiation due to the $\mathrm{Li}$ concentration gradient. For delithiation, the Li concentration on the surface of the electrode becomes zero, which leads to Li-ion diffusion out of the electrode since the $\mathrm{Li}$ concentration gradient is opposite to the lithiation process. Thus, the Li concentration decreases linearly during de-lithiation, as seen in Fig. 3a, and the remaining $\mathrm{Li}$ concentration becomes 1.22 moles Li per mole $\mathrm{Si}$ after the first lithiation-delithiation cycle, which corresponds to the rightmost Si model in Fig. 3b, having only $95 \%$ volume expansion relative to the original electrode geometry. The computed Li concentration distribution in the Si active layer during the first lithiation-delithiation cycle is described in detail in Movie S1. 
The high volume change of $\mathrm{Si}$ during the process will affect mechanically the $\mathrm{Ni}$ scaffold that supports the $\mathrm{Si}$ active layer, and this behavior can be predicted by considering the proper mechanical contact conditions as mentioned above. Fig. 4a shows the strain profiles developed in $\mathrm{Ni}$ due to the volume change of $\mathrm{Si}$ during the first lithiation-delithiation cycle. The black and red lines correspond to the computed volume average of the maximum and minimum principal strains from each element, respectively, while the blue line corresponds to one third of the computed volume average of the volumetric strain from each element. Specifically, we compute the volume average of the volumetric strain from each element in the FE model and divide by three to compare with the experimental results since the experimental strain data collected from X-ray diffraction is likely very similar to one third of the volumetric strain, which gives an "equivalent uniaxial strain”. The strains developed in the Ni scaffold during lithiation are compressive since the Si active layer expands on lithiation and is thus under net tensile strains, as seen in Fig. 4a, which indicates that the "equivalent uniaxial strain" can be replaced by the "compressive strain” in the Ni scaffold. During lithiation, Li ions diffuse into the Si active layer, thus causing an expansion of the Si which occurs isotropically. While the Si active layer expands toward to the pores, expansion of Si toward the scaffold is constrained by the Ni scaffold. This elastic resistance to the expansion of the Si active layer puts the Ni scaffold into net average compression, which increases as the Li concentration increases. The computed volume average of the compressive strain at the full lithiation point is $-0.0637 \%$ (Fig. 4a), which is similar to the measured value of $0.0767 \pm 0.002 \%$ from a previous experimental study [26]. Upon the subsequent delithiation, a rapid recovery of the compressive strain occurs, though strain is not fully 
recovered from the prelithiation state since the delithiation time is less than the lithiation time. The computed compressive strain of the Ni scaffold decreases and becomes $0.0398 \%$ after the first lithiation-delithiation cycle, which is broadly similar to the measured value of $-0.0374 \%$ from the previous experimental study [26]. After the rapid recovery, the knee in the curve at $3.8 \mathrm{~h}$ is observed, which indicates that plastic deformation might occur in the Ni scaffold since the equivalent uniaxial strain is nearly unchanged.

Plastic deformation in the Ni scaffold during lithiation can be also predicted from the computed results. Fig. 4b shows the von Mises stress distributions of the Ni scaffold under initial, full lithiation, and full de-lithiation states, which is described in detail in Movie S2. The maximum von Mises stress developed in the Ni scaffold during lithiation (about 1,200 MPa) exceeds the yield point of the $\mathrm{Ni}$ (taken as $600 \mathrm{MPa}$ ) [33] in the $4.1 \%$ of the scaffold, at regions where the Ni scaffold struts are at their thinnest, which can lead to the yielding in small regions of Ni scaffold. The von Mises stress distributions of the Ni scaffold (Fig. 4b) also indicate that the stress developed in the struts is higher than that in the nodes during (de)lithiation, thus causing a stress gradient between the nodes and struts, as seen in Fig. 4b. This phenomenon might be explained by the geometrical characteristics of opal structure. The struts are thinner than the nodes and are thus more affected by the diffusion-induced volume change of the active layer with an even thickness of $30 \mathrm{~nm}$, since stiffness is proportional to thickness. After the first lithiationdelithiation cycle, the von Mises stresses in some regions (3.2 vol. \% of the scaffold) are larger than the yield point of the $\mathrm{Ni}$, and plasticity may cause mechanical degradation of the electrode by fatigue after numerous lithiation cycles. 


\subsection{Effects of mechanical contact conditions on the developed strains in $\mathrm{Ni}$}

The calculated strains developed in the Ni scaffold due to the volume change of the Si active layer during (de)lithiation are affected by the mechanical contact conditions assumed to exist between the two phases. Fig. 5a shows the Li concentration (black) and the corresponding volumetric strain (blue) profiles in the Si active layer during the first three lithiation-delithiation cycles. As mentioned previously, 1.22 moles of Li-ions per mole Si remain in the Si active layer after the first cycle, and $\mathrm{Li}$ further accumulates in $\mathrm{Ni}$ at the end of the next two cycles, as seen in Fig. 5a.

Based on these results, strain profiles developed in the Ni scaffold during the first three cycles (Fig. 5b) are predicted using the linear pressure contact condition having no inter-penetration and slip, as explained in the supplementary material. The first lithiationdelithiation cycle behavior is identical to the on in Fig. 4a, and in the subsequent cycles, a very small amount of compressive strain accumulates in the Ni scaffold after each cycle while the magnitudes of the maximum and minimum principal strains increase gradually. The computed compressive strains of Ni scaffold after each cycle are $-0.0398,-0.0401$, and $-0.0405 \%$, respectively.

To investigate the mechanical contact effect on the strains developed in the $\mathrm{Ni}$ scaffold, two extreme contact conditions are studied: the first is a "no-interaction" condition between the Ni scaffold and Si active layer surfaces, and the other one is a "no volume change condition", where the volume of the Ni scaffold is held constant, which corresponds to incompressibility. Fig. 6a shows the strain profiles developed in the $\mathrm{Ni}$ scaffold during the first three lithiation-delithiation cycles under the no-interaction condition between the two phases. The developed strains are almost zero, indicating that 
under no-interaction condition, the volume change of the Si active layer does not transfer stresses to the Ni scaffold. Thus, physically reasonable contact conditions (such as the standard contact conditions used in the main calculation) are necessary to predict the mechanical deformation of the scaffold coated with the active layer during (de)lithiation.

For design of an effective electrode, the scaffold can be changed into a stiffer material, which is equivalent to having the outer surfaces of the scaffold pinned without affecting the volumetric expansion and contraction of the Si active layer; this corresponds to the second extreme contact condition. Based on this contact condition, the strains developed in the Ni scaffold are predicted, as seen in Fig. 6b. The computed maximum and minimum principal strains have the same orders of magnitude with the computed strains obtained from the standard contact condition (Fig. 5b), but the computed compressive strain is almost zero since the volumetric change of the Ni scaffold does not occur due to the pinned outer surfaces. Therefore, the insight (e.g. smaller strains are expected in stiffer material) gained by studying the second extreme contact condition may serve as a design guideline in fabricating practical electrodes with reliable mechanical properties.

\subsection{Comparison with in operando measured XRD data}

The computed strains are compared with XRD in-plane average crystallographic strains developed in the Ni scaffold during lithiation as measured in the Ni (200), (220) and (311) diffraction rings during the first cycle (the lattice strain analysis method is available in Ref. [26]). Fig. 7 shows a comparison of the computed and measured strain profiles in the Ni scaffold during the first lithiation-delithiation cycle, under the standard contact condition. The measured crystallographic strains are within the computed maximum and minimum principal strains during (de)lithiation, thus indicating general 
quantitative agreement between the model and experiment. Furthermore, the overall computed and measured strain evolutions match well, as described in the following. During initial lithiation, the measured strains increase linearly as the degree of lithiation increases. The differences in the slope of the strain curves and the magnitude of the compressive strains observed between Ni (200), (220) and (311) correlate linearly with their differences in stiffness (approximately 137 GPa for (200), 233 GPa for (220) and 217 GPa for (311)) [34]. Additionally, the slopes of the strain curves measured in the $\mathrm{Ni}$ (220) and (311) are similar to the computed one third volumetric strain curve. As lithiation progresses, the slopes of the experimentally measured strain curves start to depart from the red dotted line in Fig. 7 at 1.75 h, which has been interpreted in Ref. [26] as yield and plastic deformation of the Ni scaffold. This change of slope is also observed in the curves of simulated strains, and plastic deformation occurs at the red dotted line, as seen in Fig. S5. This verifies that the FE model is able to predict the strain and stress evolution of the Ni scaffold during lithiation. Upon the subsequent first delithiation, a rapid recovery of strain occurs with similar slopes for both experimental and computed strain curves between 3.3 and $3.8 \mathrm{~h}$. After the rapid recovery beyond $3.8 \mathrm{~h}$, a change in the slope of the strain curve is observed in the both experimental and computed strain curves, which indicates that the plastic deformation might occur in the Ni scaffold since the plastic deformation does not have any volume change. A comparison of the computed and measured strain profiles in the Ni scaffold during the first three lithiation-delithiation cycles is described in the supplementary material (Fig. S6).

Overall, the computed strain profiles agree with the measured data obtained from in operando XRD, which is a powerful method for characterizing transient phenomena in 
battery materials. The good quantitative and qualitative match in strain history over multiple (de)lithiation cycles is the first step towards understanding more complex issues such as fracture and mechanical degradation of the LIB anodes, for which damage initiation and evolution will need to be considered both numerically (using much finer meshing) and experimentally (via spatially resolved measurements)..

\subsection{Plastic strain behavior in Si}

Based on the above validation of the FE model in the Ni scaffold, we investigate plastic deformation in the Si active layer during first lithiation-delithiation cycle, for which no experimental data are available. Fig. 8 shows the computed volume-averaged von Mises (equivalent) plastic strain from each element in the Si active layer during the first lithiation-delithiation cycle. The onset of the plasticity in the Si active layer starts at about $0.3 \mathrm{~h}$, which indicates that plastic deformation occurs first in the lithiated $\mathrm{Si}$, then the Ni scaffold, comparing with Fig. S5 in the supplementary material. At the onset of the plasticity, plastic strain is concentrated at some regions (highlighted with black circles in

Fig. 8). Given that the volume expansion of the Si active layer is hindered geometrically upon lithiation, these regions of the $\mathrm{Si}$ active layer are the most likely to undergo mechanical damage and cracking. The equivalent plastic strain increases linearly from the onset of plasticity at $0.3 \mathrm{~h}$ to the full lithiation state, and the computed equivalent plastic strain at the full lithiation is 0.492 . Plastic strain is still concentrated at some regions (highlighted with black ellipse in Fig. 8). Upon the onset of the subsequent first delithiation cycle, plastic strain does not immediately increase since elastic recovery occurs first, due to the out-diffusion of $\mathrm{Li}$ atoms. The equivalent plastic strain increases once again from $3.8 \mathrm{~h}$. The large build-up of plasticity during the first lithiation and 
increase in the equivalent plastic strain during the first delithiation implies that the $\mathrm{Si}$ experiences reverse plasticity from $3.8 \mathrm{~h}$ since we consider the strain hardening behavior of the Si, as seen in Fig. S2. The computed equivalent plastic strain distribution during the first lithiation-delithiation is described in detail in Movie S3. The FE model enables detailed examination of the distribution and history of strains, as well as the degree of plasticity present in the Si active layer upon lithiation, which cannot be measured in in operando XRD since the Si active layer is not crystalline. This illustrates the importance of FE model which can predict overall mechanical phenomenon of anodes.

\section{Conclusions}

We study strains and stresses created by constrained expansion/contraction during (de)lithiation of a Si active layer deposited on a Ni scaffold. We demonstrate here that strain analysis can be achieved within a sequentially coupled diffusion-stress FE model that incorporates mechanical contact between the active Si and the passive Ni phases, complex geometry (inverse opal), and mechanical properties for both phases. We compare our numerical results for strains with prior in operando XRD-based strain experimental data. Once verified, the FE approach allows for the analysis of the mechanical behavior of LIB anodes and opens the door to understanding and predicting the mechanical degradation of electrodes due to the lithiation-induced volume change.

The very high volume change (up to $310 \%$ ) of the Si active layer during lithiation causes the mechanical deformation of the Ni scaffold, which results in the computed compressive strain of $0.0637 \%$ in our model at the full lithiation. Upon subsequent delithiation, a rapid recovery of compressive strain occurs, resulting in computed 
compressive strain of $0.0398 \%$ in the $\mathrm{Ni}$ scaffold after the first lithiation-delithiation cycle. A von Mises stress greater than the yield point of the Ni scaffold is developed in some regions of the Ni scaffold, indicating that plasticity is occurring, and the stresses are concentrated more in the struts than the nodes, thus causing a stress gradient in the scaffold.

From case studies, physically reasonable contact conditions are necessary to predict the mechanical deformation of the scaffold coated with the active layer during (de)lithiation. The computed strain profiles are compared with the measured X-ray data. The measured strains are within the computed maximum and minimum principal strains, and, during (dis)charging of the battery, the time evolution of the measured in-plane average crystallographic strain curves are similar to the computed compressive strain curve. Finally, the plastic strain in the Si active layer is computed, which enables the prediction of the onset of the plasticity, the plastic strain concentrated regions, and the degree of plasticity in the Si active layer.

Once verified, the FE approach allows analyzing the mechanical behavior of LIB anodes and opens the door to understanding and predicting the mechanical degradation of electrodes due to the lithiation-induced volume change.

\section{Experimental Section}

Electrode Preparation: The preparation of bicontinuous inverse opal silicon battery anodes is described in detail in Glazer et al. and elsewhere [17, 26, 31]. Briefly, to prepare $\sim 10 \mu \mathrm{m}$ thick opal nickel template structures, $500 \mathrm{~nm}$ diameter polystyrene spheres (PS), are self-assembled on a gold-coated $500 \mu \mathrm{m}$ thick single crystal silicon (100) 
substrate (coated with its native oxide). The PS sphere arrangement is then heated at a temperature near the glass-transition temperature of the PS to strengthen the opal template structure, after which $\mathrm{Ni}$ is electrodeposited into the void space of the PS opal template. The PS template is then removed with tetrahydrofuran, exposing a Ni inverse opal scaffold with narrow struts interconnected between spherical voids. The scaffold is then electrochemically etched to increase the window size between voids and the porosity of the structure. Finally, a thin $(\sim 30 \mathrm{~nm})$ film of amorphous $\mathrm{Si}$ is deposited onto the $\mathrm{Ni}$ scaffold through chemical vapor deposition (CVD) at $400{ }^{\circ} \mathrm{C}$ using disilane as the $\mathrm{Si}$ source.

Electrochemical Cell Fabrication: Electrochemical pouch cells were prepared to perform in operando diffraction measurements of the Si-coated Ni scaffold as described in Glazer et al. [26]. The Si anode was attached to an external electrical contact via conductive carbon tape attached to the gold coating on the Si substrate. A Celgard 2500 polypropylene separator was placed between the anode and a $380 \mu \mathrm{m}$ lithium foil counter electrode, with the whole assembly bathed in a non-aqueous electrolyte and sealed in a polyamide-coated aluminum pouch inside of an argon-filled glove box.

In Operando X-ray Diffraction Measurements: Diffraction experiments were performed at the Advanced Photon Source, at Argonne National Laboratory, using the beam line station 1-BM-C as described in Glazer et al. [26]. A monochromatic X-ray with an energy of $20.25 \mathrm{keV}(\lambda=0.061234 \mathrm{~nm})$ and a $300 \mu \mathrm{m}$ beam diameter shined on the center of the sample, aligned such that all elements of the electrochemical pouch cell, including the Ni scaffold, were nearly normal to the beam direction. Diffraction patterns were recorded in a transmission geometry, and taken every five minutes with a 30s 
exposure time with two-dimensional position sensitive area detector. The sample was slightly tilted to ensure the single crystal Si substrate peaks were out of the Bragg condition, to reduce any strong diffraction spots onto the detector. A diffraction pattern from a reference powder sample of $\mathrm{LaB}_{6}(1 \mathrm{~mm}$ kapton capillary tube filled with NIST Standard Reference Material 660b) and the software FIT2D [35] was used to provide a calibration standard for the experimental setup. The electrochemical cell cycling parameters are described in Glazer et al. [26]. The strain analysis methods employed in the interpretation of the in operando XRD measurements are briefly described in the supplemental material and discussed in detail in Glazer et al. [26].

\section{Appendix A. Supplementary material}

Supplementary material is available at

\section{Acknowledgements}

This research was supported by the U.S. Department of Energy, Office of Basic Energy Sciences, under Contract No. DE-AC02-06CH11357 and Dongbu Cultural Foundation. H.N.H. was supported by Basic Science Research Program through the National Research Foundation of Korea (NRF) funded by the Ministry of Science, ICT and Future Planning (NRF-2013R1A2A2A01008806). The authors thank, Drs. Jonathan Almer and John Okasinski (Argonne National Laboratory) for useful discussions.

\section{References}


[1] M.N. Obrovac, L. Christensen. Structural changes in silicon anodes during lithium insertion/extraction, Electrochemical and Solid State Letters 7 (2004) A93-A96.

[2] P. Arora, R.E. White, M. Doyle. Capacity fade mechanisms and side reactions in lithium-ion batteries, Journal of the Electrochemical Society 145 (1998) 3647-3667.

[3] J.W. Wang, X.H. Liu, K.J. Zhao, A. Palmer, E. Patten, D. Burton, S.X. Mao, Z.G. Suo, J.Y. Huang. Sandwich-Lithiation and Longitudinal Crack in Amorphous Silicon Coated on Carbon Nanofibers, Acs Nano 6 (2012) 9158-9167.

[4] M.T. McDowell, I. Ryu, S.W. Lee, C.M. Wang, W.D. Nix, Y. Cui. Studying the Kinetics of Crystalline Silicon Nanoparticle Lithiation with In Situ Transmission Electron Microscopy, Advanced Materials 24 (2012) 6034-+.

[5] M.T. McDowell, S.W. Lee, J.T. Harris, B.A. Korgel, C.M. Wang, W.D. Nix, Y. Cui. In Situ TEM of Two-Phase Lithiation of Amorphous Silicon Nanospheres, Nano Letters 13 (2013) 758-764.

[6] M. Gu, Y. Li, X.L. Li, S.Y. Hu, X.W. Zhang, W. Xu, S. Thevuthasan, D.R. Baer, J.G. Zhang, J. Liu, C.M. Wang. In Situ TEM Study of Lithiation Behavior of Silicon Nanoparticles Attached to and Embedded in a Carbon Matrix, Acs Nano 6 (2012) 84398447.

[7] L.Y. Beaulieu, T.D. Hatchard, A. Bonakdarpour, M.D. Fleischauer, J.R. Dahn. Reaction of Li with alloy thin films studied by in situ AFM, Journal of the Electrochemical Society 150 (2003) A1457-A1464.

[8] V.A. Sethuraman, M.J. Chon, M. Shimshak, V. Srinivasan, P.R. Guduru. In situ measurements of stress evolution in silicon thin films during electrochemical lithiation and delithiation, Journal of Power Sources 195 (2010) 5062-5066.

[9] V.A. Sethuraman, M.J. Chon, M. Shimshak, N. Van Winkle, P.R. Guduru. In situ measurement of biaxial modulus of Si anode for Li-ion batteries, Electrochem Commun 12 (2010) 1614-1617.

[10] X.Q. Yang, J. McBreen, W.S. Yoon, M. Yoshio, H.Y. Wang, K. Fukuda, T. Umeno. Structural studies of the new carbon-coated silicon anode materials using synchrotron-based in situ XRD, Electrochemistry Communications 4 (2002) 893-897.

[11] T.D. Hatchard, J.R. Dahn. In situ XRD and electrochemical study of the reaction of lithium with amorphous silicon, Journal of the Electrochemical Society 151 (2004) A838-A842.

[12] J. Li, J.R. Dahn. An in situ X-ray diffraction study of the reaction of Li with crystalline Si, Journal of the Electrochemical Society 154 (2007) A156-A161.

[13] S. Misra, N. Liu, J. Nelson, S.S. Hong, Y. Cui, M.F. Toney. In Situ X-ray Diffraction Studies of (De)lithiation Mechanism in Silicon Nanowire Anodes, Acs Nano 6 (2012) 5465-5473.

[14] F. Wang, L.J. Wu, B. Key, X.Q. Yang, C.P. Grey, Y.M. Zhu, J. Graetz. Electrochemical Reaction of Lithium with Nanostructured Silicon Anodes: A Study by In-Situ Synchrotron X-Ray Diffraction and Electron Energy-Loss Spectroscopy, Advanced Energy Materials 3 (2013) 1324-1331.

[15] A.F. Bower, P.R. Guduru. A simple finite element model of diffusion, finite deformation, plasticity and fracture in lithium ion insertion electrode materials, Modelling and Simulation in Materials Science and Engineering 20 (2012). 
[16] M. Liu. Finite element analysis of lithium insertion-induced expansion of a silicon thin film on a rigid substrate under potentiostatic operation, Journal of Power Sources 275 (2015) 760-768.

[17] H.G. Zhang, P.V. Braun. Three-Dimensional Metal Scaffold Supported Bicontinuous Silicon Battery Anodes, Nano Letters 12 (2012) 2778-2783.

[18] I.C. Noyan, T.C. Huang, B.R. York. Residual-Stress Strain Analysis in ThinFilms by X-Ray-Diffraction, Critical Reviews in Solid State and Materials Sciences 20 (1995) 125-177.

[19] A. Wanner, D.C. Dunand. Synchrotron X-ray study of bulk lattice strains in externally loaded Cu-Mo composites, Metallurgical and Materials Transactions aPhysical Metallurgy and Materials Science 31 (2000) 2949-2962.

[20] D.K. Balch, E. Ustundag, D.C. Dunand. Diffraction strain measurements in a partially crystallized bulk metallic glass composite containing ductile particles, Journal of Non-Crystalline Solids 317 (2003) 176-180.

[21] J.D. Almer, S.R. Stock. Internal strains and stresses measured in cortical bone via high-energy X-ray diffraction, Journal of Structural Biology 152 (2005) 14-27.

[22] M.R. Daymond, M.L. Young, J.D. Almer, D.C. Dunand. Strain and texture evolution during mechanical loading of a crack tip in martensitic shape-memory NiTi, Acta Materialia 55 (2007) 3929-3942.

[23] M.L. Young, J. DeFouw, J.D. Almer, D.C. Dunand. Load partitioning during compressive loading of a Mg/MgB2 composite, Acta Materialia 55 (2007) 3467-3478.

[24] A.C. Deymier-Black, J.D. Almer, S.R. Stock, D.R. Haeffner, D.C. Dunand. Synchrotron X-ray diffraction study of load partitioning during elastic deformation of bovine dentin, Acta Biomaterialia 6 (2010) 2172-2180.

[25] N.S. Rossini, M. Dassisti, K.Y. Benyounis, A.G. Olabi. Methods of measuring residual stresses in components, Materials \& Design 35 (2012) 572-588.

[26] M.P.B. Glazer, J. Cho, J. Almer, J. Okasinski, P.V. Braun, D.C. Dunand. In Operando Strain Measurement of Bicontinuous Silicon-Coated Nickel Inverse Opal Anodes for Li-Ion Batteries, Advanced Energy Materials (2015).

[27] S.B. Lee, W.L. Wang, J.R. Chen. Diffusion-induced stresses in a hollow cylinder: Constant surface stresses, Materials Chemistry and Physics 64 (2000) 123-130.

[28] F.Q. Yang. Insertion-induced expansion of a thin film on a rigid substrate, Journal of Power Sources 241 (2013) 146-149.

[29] R. Purkayastha, R. McMeeking. A parameter study of intercalation of lithium into storage particles in a lithium-ion battery, Comp Mater Sci 80 (2013) 2-14.

[30] R.M. McMeeking, R. Purkayastha. The role of solid mechanics in electrochemical energy systems such as lithium-ion batteries, Proc Iutam 10 (2014) 294-306.

[31] H.G. Zhang, X.D. Yu, P.V. Braun. Three-dimensional bicontinuous ultrafastcharge and -discharge bulk battery electrodes, Nature Nanotechnology 6 (2011) 277-281.

[32] I. Ryu, J.W. Choi, Y. Cui, W.D. Nix. Size-dependent fracture of Si nanowire battery anodes, Journal of the Mechanics and Physics of Solids 59 (2011) 1717-1730.

[33] F. Ebrahimi, G.R. Bourne, M.S. Kelly, T.E. Matthews. Mechanical properties of nanocrystalline nickel produced by electrodeposition, Nanostructured Materials 11 (1999) 343-350.

[34] T.H. Courtney. Mechanical behavior of materials. 2nd ed., McGraw Hill, Boston, 2000. 
[35] A.P. Hammersley, S.O. Svensson, A. Thompson, H. Graafsma, A. Kvick, J.P. Moy. Calibration and Correction of Distortions in 2-Dimensional Detector Systems, Review of Scientific Instruments 66 (1995) 2729-2733. 


\section{Figures}

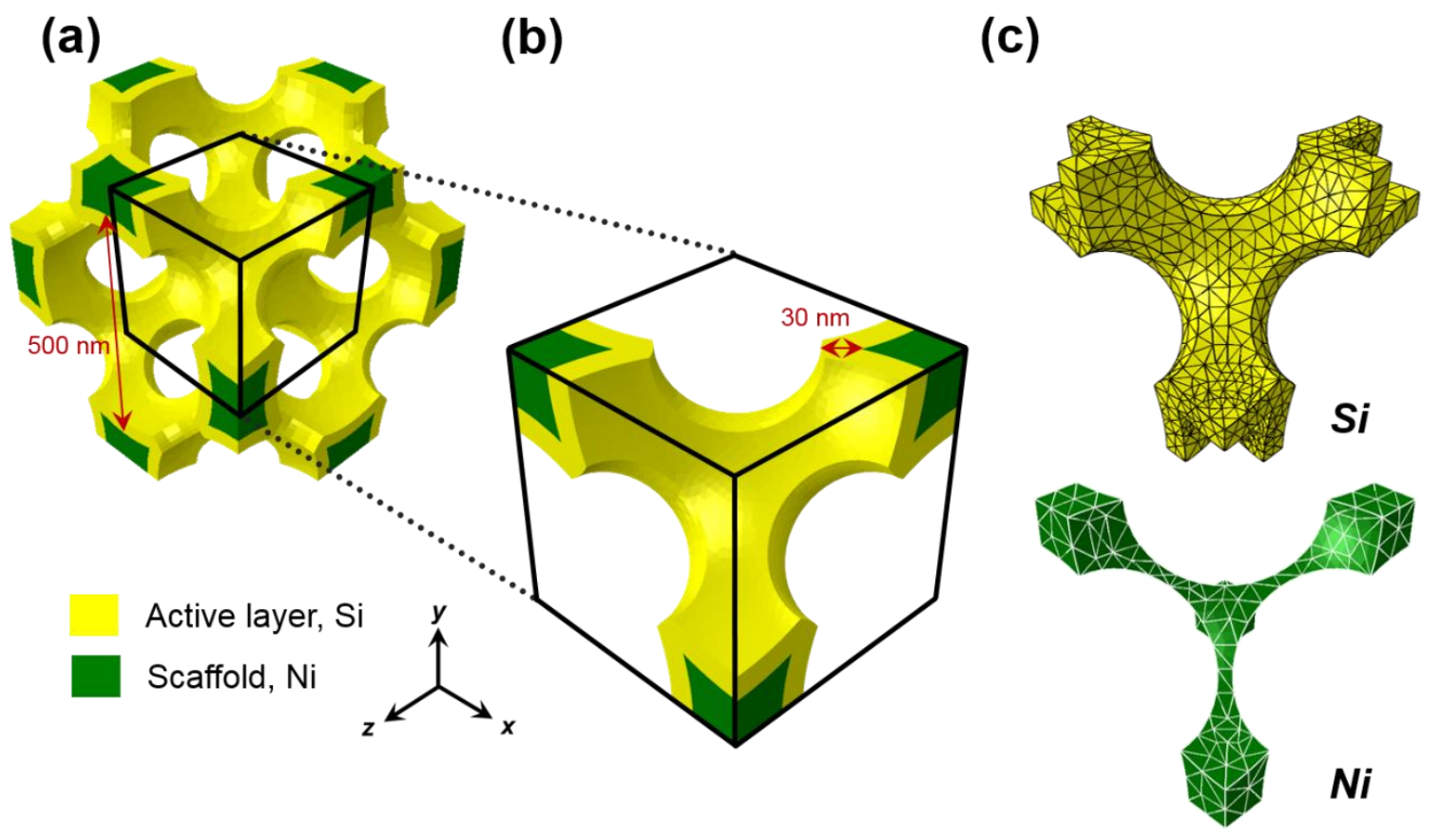

Fig. 1. 3D structure and mesh systems of the Si-coated Ni inverse opal electrode: (a) the Ni scaffold with a pore size of $500 \mathrm{~nm}$ coated with a $30 \mathrm{~nm}$ thick Si active layer, (b) the representative volume element (RVE) used in the FE model, and (c) the 3D mesh systems of the Si active layer and Ni scaffold. The Si and Ni structures have 9,063, and 696 tetrahedral elements, respectively. 
(a)

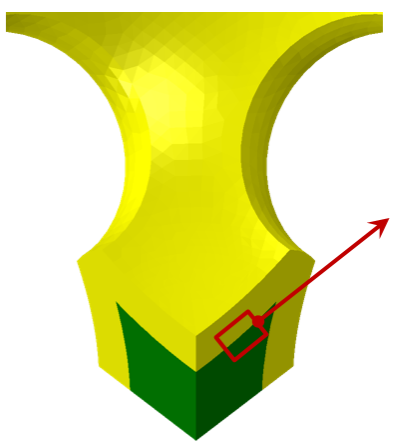

(b)

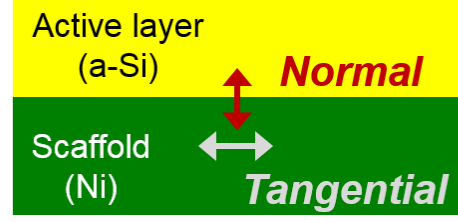

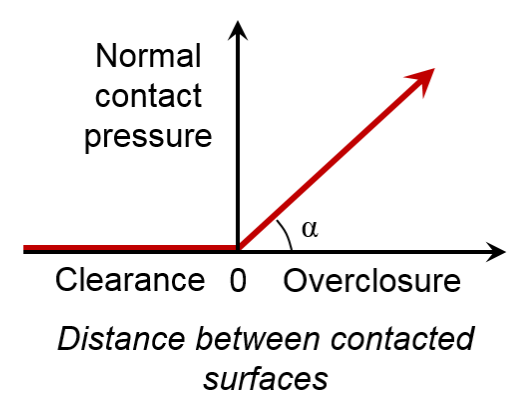

Fig. 2. Schematic diagrams of the contact condition between the Si and Ni phases: (a) the normal and tangential directions used to describe contact behavior and (b) the normal contact pressure as a function of the distance between the $\mathrm{Si}$ and $\mathrm{Ni}$ phases, corresponding to a linear contact condition. $\alpha$ corresponds to the slope of the change in normal contact pressure upon over-closure. 
(a)

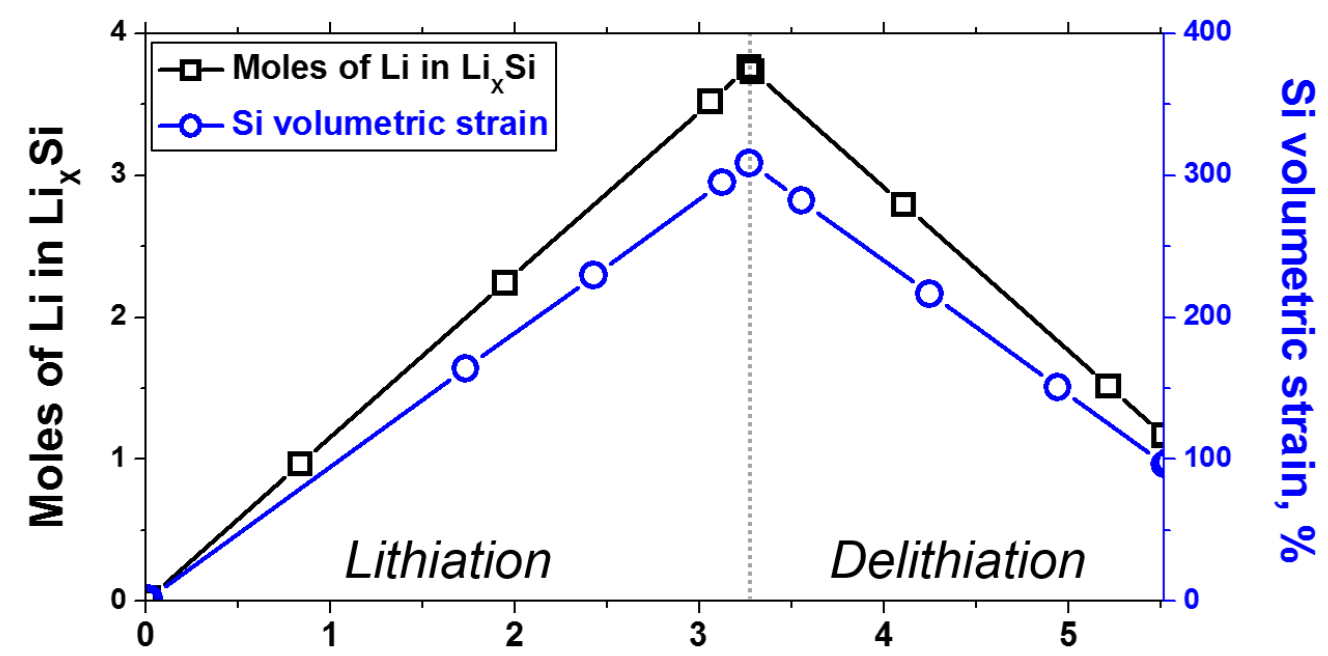

(b)

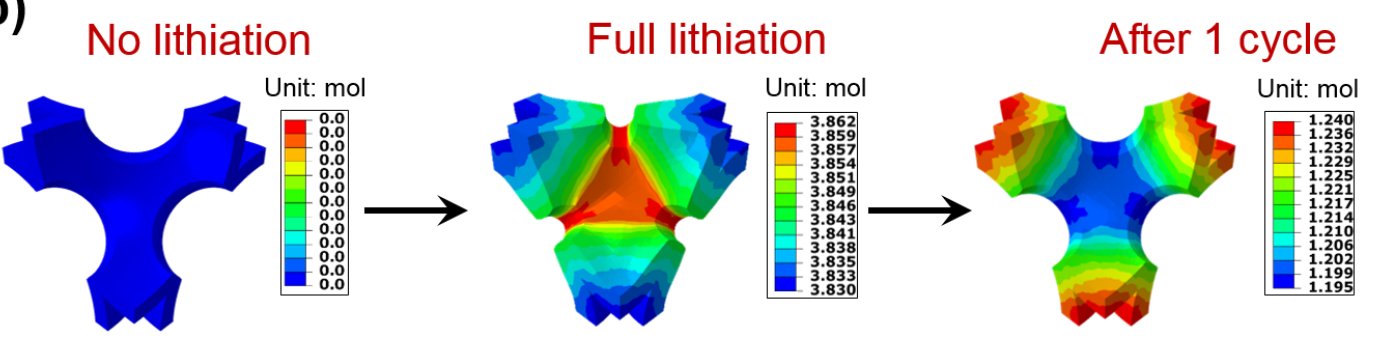

Fig. 3. (a) The computed Li concentration (black) and corresponding volumetric strain profile (blue) in the Si active layer during the first lithiation-delithiation cycle, and (b) the computed Li concentration distributions in the Si active layer at the initial, full lithiation, and full de-lithiation states, which is described in detail in Movie S1. The contours show isoconcentration surfaces of $\mathrm{Li}$ in the $\mathrm{Si}$ active layer. 
(a)

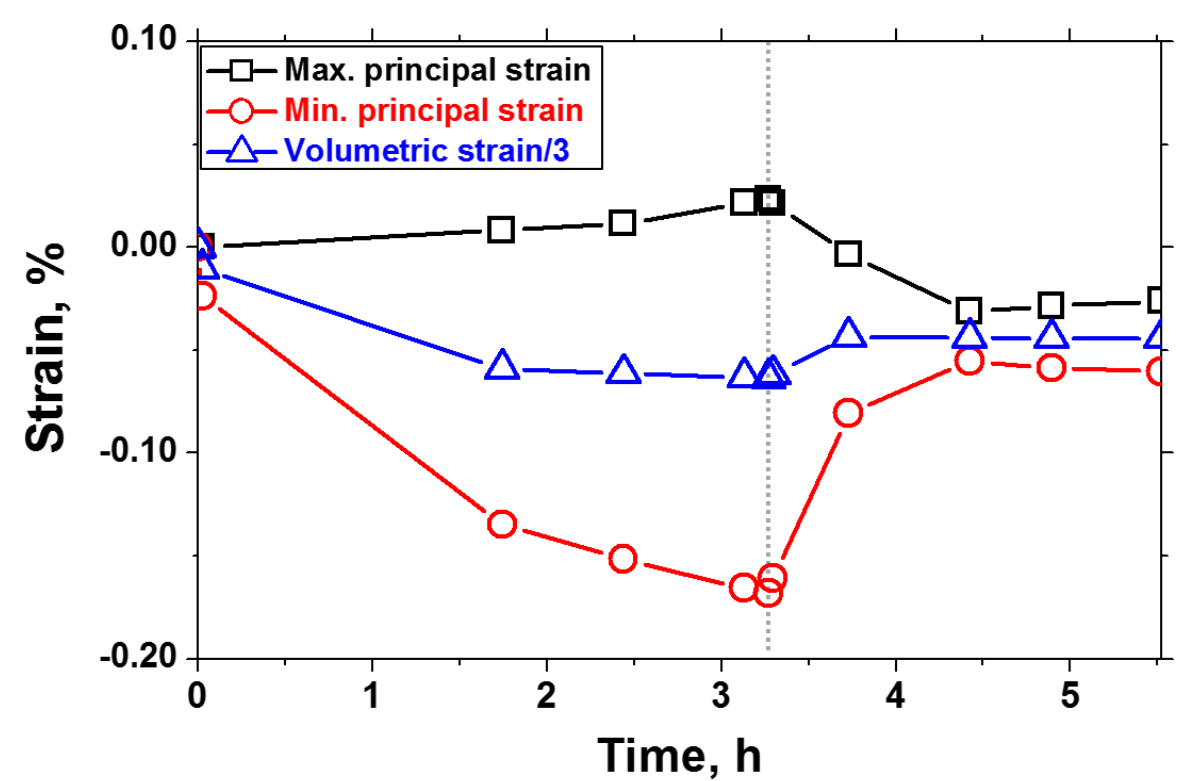

(b) No lithiation

Full lithiation

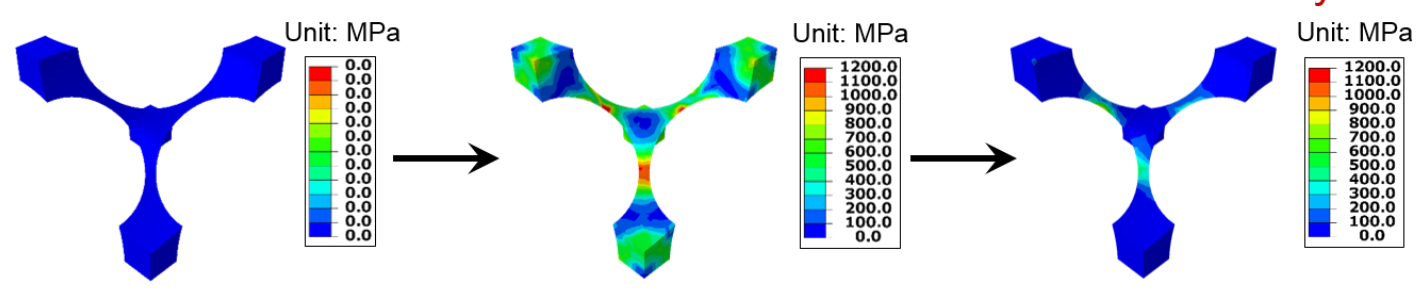

Fig. 4. (a) Computed strain profiles developed in the Ni scaffold due to the volume change of the Si during the first lithiation-delithiation cycle. The black and red lines correspond to the volume average of the maximum and minimum principal strains from each element, respectively, while the blue line corresponds to one third of the volume average of the volumetric strain from each element. (b) The von Mises stress distributions of the Ni scaffold at the initial, full lithiation (end of first half-cycle), and end of first cycle, in the delithiated state, which is described in detail in Movie S2. The color contours correspond surfaces of equivalent stress. 
(a)
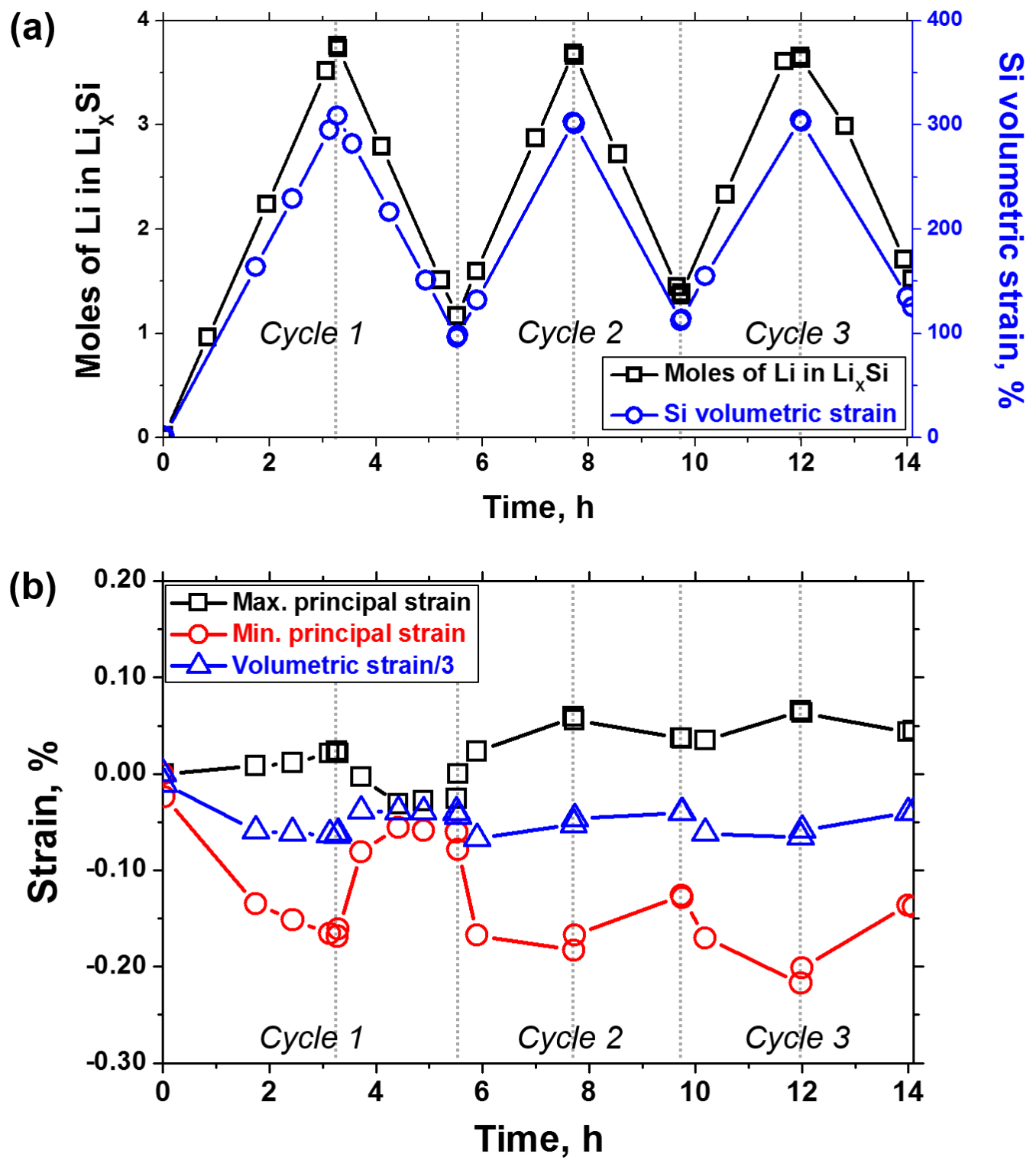

Fig. 5. (a) Li concentration (black) and the corresponding volumetric strain (blue) evolution in the Si active layer during the first three lithiation-delithiation cycles and (b) strain profiles developed in the Ni scaffold due to the volume change of the Si during these cycles. The black and red lines correspond to the volume average of the maximum and minimum principal strains from each element, respectively, while the blue line 
corresponds to one third of the volume average of the volumetric strain from each element (as in Fig. 4). 
(a)

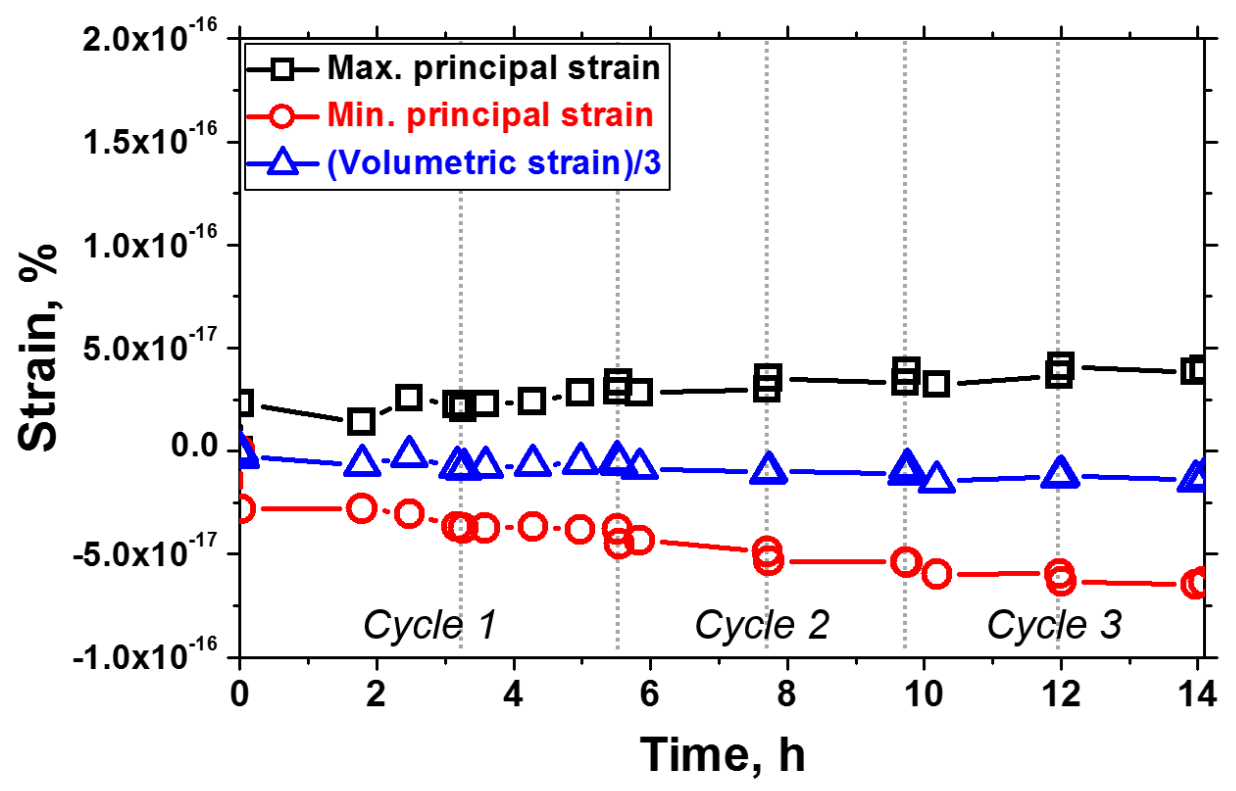

(b)

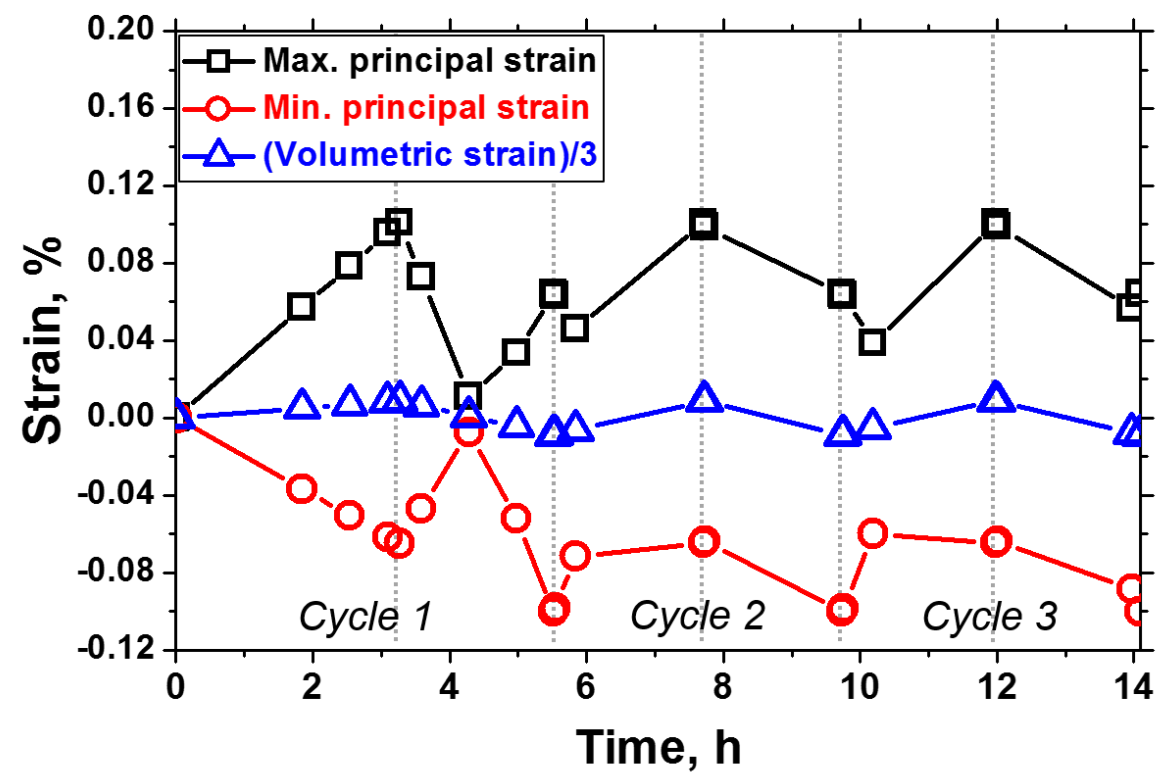

Fig. 6. Computed strains developed in the Ni scaffold during the first three lithiationdelithiation cycles under two extreme contact conditions: (a) no-interaction condition between the $\mathrm{Ni}$ and $\mathrm{Si}$ and (b) no-volume change condition, where no volume change is allowed in the Ni scaffold while keeping volumetric strain of the $\mathrm{Si}$ active layer. The black and red lines correspond to the volume average of the maximum and minimum 
principal strains, respectively, while the blue line corresponds to one third of the volume average of the volumetric strain from each element (as in Fig. 4 and Fig. 5). 


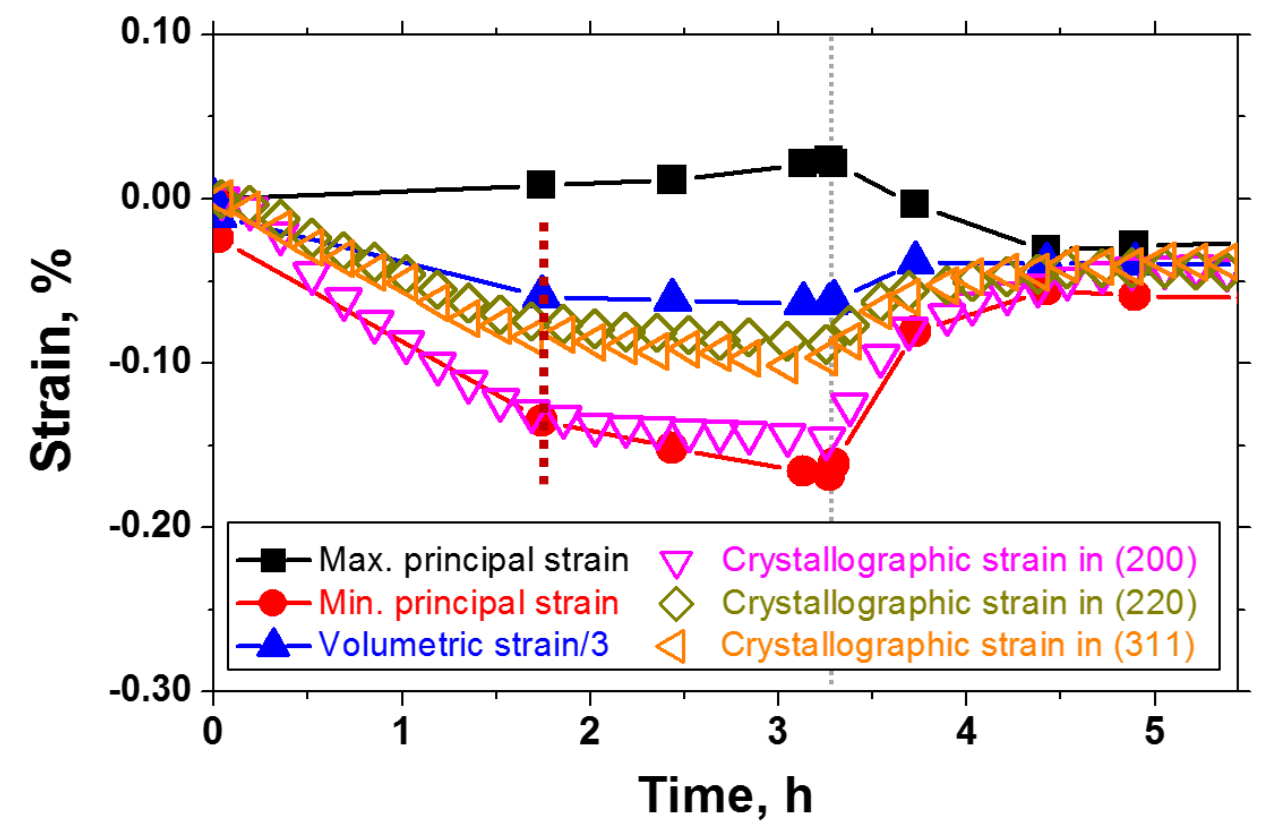

Fig. 7. Comparison of the simulated and measured strain profiles in the $\mathrm{Ni}$ scaffold during the first lithiation-delithiation cycle. The solid lines indicate the simulated strains (the black and red lines correspond to the maximum and minimum principal strains, respectively, while the blue line corresponds to one third of the volumetric strain) while the markers represent strain data measured by XRD by Glazer et al. [26]: in-plane average crystallographic strains measured from Ni (200), (220), and (311) diffraction peaks (magenta downward triangle, green diamond, and orange left pointing triangle, respectively). The red dotted vertical line indicates the location of the change in strain rate from a linear strain during lithiation. The sizes of the markers for the experimental data are larger than the uncertainty of the measured strains. 


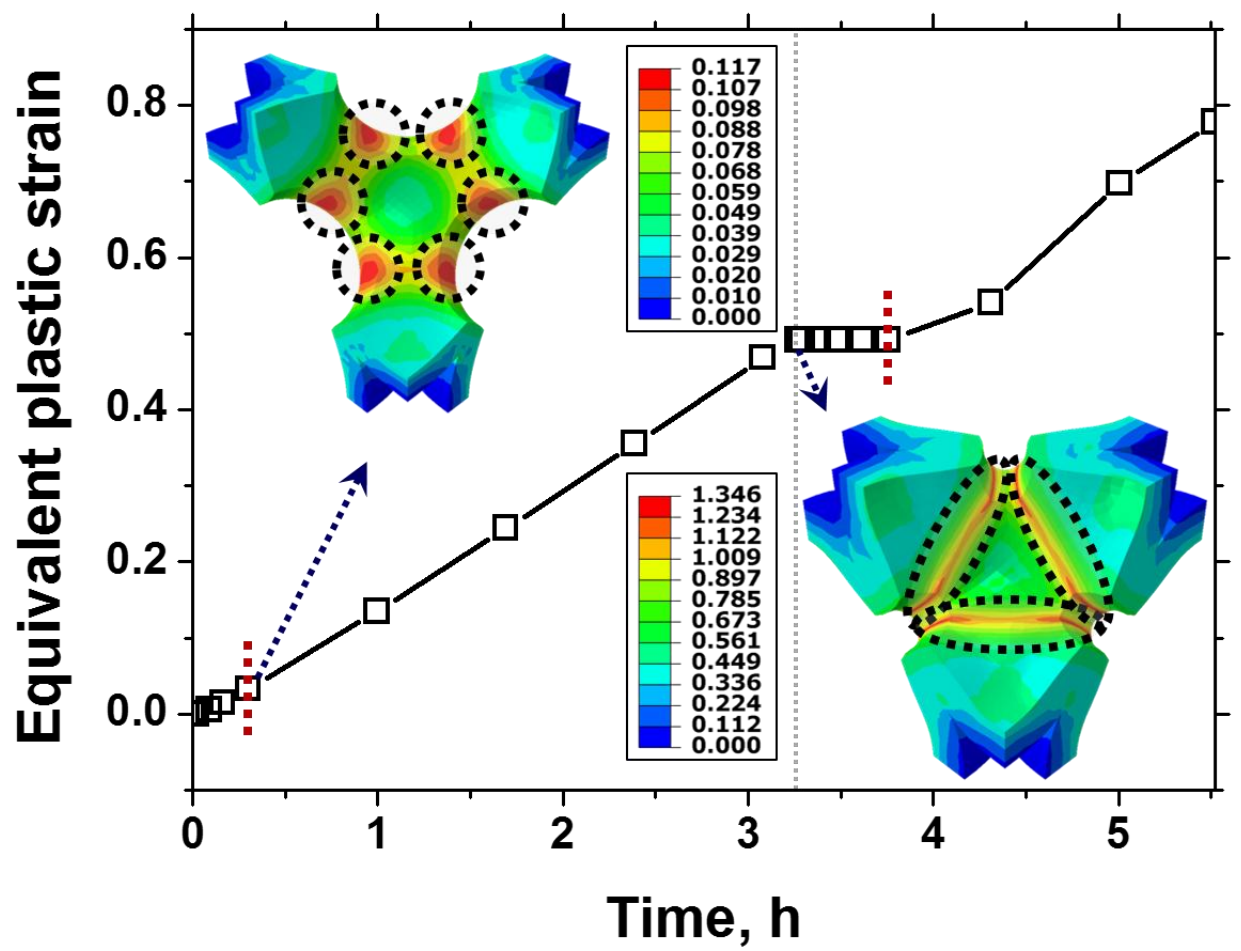

Fig. 8. Computed equivalent plastic strain profile in the $\mathrm{Si}$ active layer during the first lithiation-delithiation cycle and the equivalent plastic strain distribution of the Si active layer at the onset of the plasticity and the full lithiation with the color contours corresponding surfaces of equivalent strain. The red dotted lines indicate the locations of the onset of the plasticity during the first lithiation-delithiation, respectively. The equivalent plastic strain distribution of the $\mathrm{Si}$ active layer during the first lithiationdelithiation cycle is described in detail in Movie S3. 


\section{Tables}

Table 1. Material properties and parameter values used in the model.

\begin{tabular}{lc}
\hline Property/Parameter & Value \\
\hline Diffusivity of Li in $\mathrm{Si}(\mathrm{D})$ & $2 \mathrm{~nm}^{2} / \mathrm{sec}[30]$ \\
Solubility of $\mathrm{Li}$ in $\mathrm{Si}\left(\mathrm{c}_{\mathrm{max}}\right)$ & $53.398 \mathrm{atoms} / \mathrm{nm}^{3}[30]$ \\
Density of $\mathrm{Si}\left(\rho_{\mathrm{Si}}\right)$ & $2329 \mathrm{~kg} / \mathrm{m}^{3}[15]$ \\
Density of $\mathrm{Ni}\left(\rho_{\mathrm{Ni}}\right)$ & $8908 \mathrm{~kg} / \mathrm{m}^{3}[33]$ \\
Young's modulus of $\mathrm{Si}\left(\mathrm{E}_{\mathrm{Si}}\right)$ & $80 \mathrm{GPa}[30]$ \\
Young's modulus of $\mathrm{Ni}\left(\mathrm{E}_{\mathrm{Ni}}\right)$ & $200 \mathrm{GPa}[33]$ \\
Poisson's ratio of $\mathrm{Si}\left(v_{\mathrm{Si}}\right)$ & $0.22[30]$ \\
Poisson's ratio of $\mathrm{Ni}\left(v_{\mathrm{Ni}}\right)$ & $0.31[33]$ \\
Volumetric expansion of $\mathrm{Si}$ with Li concentration $(\Omega)$ & $0.82 \mathrm{~m} / \mathrm{mol}[34]$ \\
Plastic properties of $\mathrm{Si}$ & $\mathrm{Sethuraman}$ et al. $[8]^{*}$ \\
Plastic properties of $\mathrm{Ni}$ & Ebrahimi et al. $[31]^{*}$ \\
Specific capacity of $\mathrm{Si}\left(\mathrm{C}_{\mathrm{Si}}\right)$ & $4.2 \mathrm{Ah} / \mathrm{g} \mathrm{[30]}$ \\
Slope of normal contact pressure $(\alpha)$ & $1.2 \mathrm{E}_{\mathrm{Ni}}$ \\
Temperature $(\mathrm{T})$ & $298 \mathrm{~K}$ \\
\hline
\end{tabular}

*Stress-strain curves of Si and Ni are reproduced in Fig. S2. 
Graphical Abstract
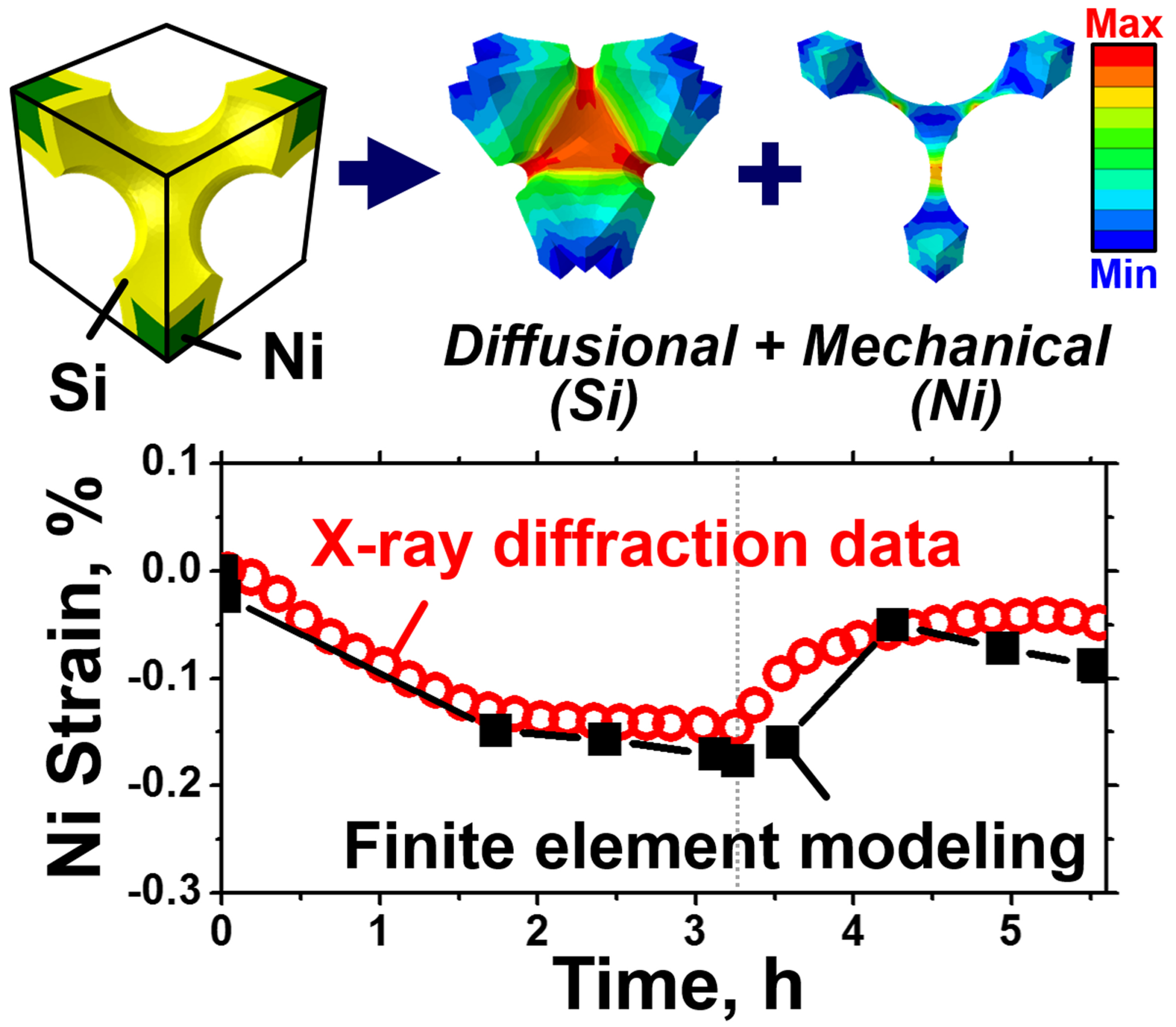